PROCEEDINGS OF THE

AMERICAN MATHEMATICAL SOCIETY

Volume 137, Number 5, May 2009, Pages 1663-1676

S 0002-9939(08)09374-X

Article electronically published on December 12, 2008

\title{
LEONHARD EULER AND A $q$-ANALOGUE OF THE LOGARITHM
}

\author{
ERIK KOELINK AND WALTER VAN ASSCHE \\ (Communicated by Peter A. Clarkson)
}

On the 300th anniversary of Euler's birth

\begin{abstract}
We study a $q$-logarithm which was introduced by Euler and give some of its properties. This $q$-logarithm has not received much attention in the recent literature. We derive basic properties, some of which were already given by Euler in a 1751 paper and in a 1734 letter to Daniel Bernoulli. The corresponding $q$-analogue of the dilogarithm is introduced. The relation to the values at 1 and 2 of a $q$-analogue of the zeta function is given. We briefly describe some other $q$-logarithms that have appeared in the recent literature.
\end{abstract}

\section{INTRODUCTION}

In a paper from 1751, Leonhard Euler (1707-1783) introduced the series [8, §6]

$$
s=\sum_{k=1}^{\infty} \frac{(1-x)(1-x / a) \cdots\left(1-x / a^{k-1}\right)}{1-a^{k}} .
$$

We will take $q=1 / a$. Then this series is convergent for $|q|<1$ and $x \in \mathbb{C}$. In this paper we will assume $0<q<1$. Then this becomes

$$
S_{q}(x)=-\sum_{k=1}^{\infty} \frac{q^{k}}{1-q^{k}}(x ; q)_{k},
$$

where $(x ; q)_{0}=1,(x ; q)_{k}=(1-x)(1-x q) \cdots\left(1-x q^{k-1}\right)$. This can be written as a basic hypergeometric series

$$
S_{q}(x)=-\frac{q(1-x)}{1-q}{ }_{3} \phi_{2}\left(\begin{array}{c}
q, q, q x \\
q^{2}, 0
\end{array} ; q, q\right) .
$$

Euler had come across this series much earlier in an attempt to interpolate the logarithm at powers $a^{k}$ (or $q^{-k}$ ); see, e.g., Gautschi's comment 11 discussing Euler's letter to Daniel Bernoulli where Euler introduced the function for $a=10$. Euler was aware that this interpolation did not work very well; see [11, §§3-4]. The function in (1.2) does not seem to appear in the recent literature, even though it has some nice properties. We will prove some of its properties, some already obtained

Received by the editors March 6, 2007.

2000 Mathematics Subject Classification. Primary 33B30, 33E30.

The second author was supported by research grant OT/04/21 of Katholieke Universiteit Leuven, research project G.0455.04 of FWO-Vlaanderen, and INTAS research network 03-51-6637. 
by Euler [8], and indicate why this should be called a $q$-analogue of the logarithm. A first reason is that for $0<q<1$,

$$
\lim _{q \rightarrow 1}(1-q) S_{q}(x)=-\sum_{k=1}^{\infty} \lim _{q \rightarrow 1} q^{k} \frac{1-q}{1-q^{k}}(x ; q)_{k}=-\sum_{k=1}^{\infty} \frac{(1-x)^{k}}{k}=\log x,
$$

which is only a formal limit transition, since interchanging limit and sum seems hard to justify.

In Sections 20 we study this $q$-analogue of the logarithm more closely. In particular, we reprove some of Euler's results. Then we go on to extend the definition in Section 4. Finally, we study the corresponding $q$-analogue of the dilogarithm in Section 5. It involves also the values at 1 and 2 of a $q$-analogue of the $\zeta$-function. We give a (incomplete) list of some other $q$-analogues of the logarithm appearing in the literature in Section 6. The purpose of this note is to draw attention to the $q$-analogues of the logarithm, dilogarithm and $\zeta$-function for which we expect many interesting results remain to be discovered.

Many results in this paper use the $q$-binomial theorem [10, §1.3], [1, §10.2]

$$
\frac{(a x ; q)_{\infty}}{(x ; q)_{\infty}}=\sum_{j=0}^{\infty} \frac{(a ; q)_{j}}{(q ; q)_{j}} x^{j}, \quad|x|<1 .
$$

We also use the $q$-exponential functions [10, p. 9], [1, p. 492]

$$
\begin{aligned}
& e_{q}(z)=\frac{1}{(z ; q)_{\infty}}=\sum_{n=0}^{\infty} \frac{z^{n}}{(q ; q)_{n}}, \quad|z|<1, \\
& E_{q}(z)=(-z ; q)_{\infty}=\sum_{n=0}^{\infty} \frac{q^{n(n-1) / 2}}{(q ; q)_{n}} z^{n} .
\end{aligned}
$$

\section{The $q$-LOGARITHM AS AN ENTIRE FUNCTION}

First of all we will show that the function $S_{q}$ in (1.2) is an entire function, and as such it is a nicer function than the logarithm, which has a cut along the negative real axis.

Property 2.1. The function $S_{q}$ defined in (1.2) is an entire function of order zero.

Proof. For $k \in \mathbb{N}$ the $q$-Pochhammer $(z ; q)_{k}$ is a polynomial of degree $k$ with zeros at $1,1 / q, \ldots, 1 / q^{k-1}$. For $|z| \leq r$ we have the simple bound

$$
\left|(z ; q)_{k}\right| \leq(1+r)(1+r|q|) \cdots\left(1+r|q|^{k-1}\right)=(-r ;|q|)_{k}<(-r ;|q|)_{\infty},
$$

and hence the partial sums are uniformly bounded on the ball $|z| \leq r$ :

$$
\left|-\sum_{k=1}^{n} \frac{q^{k}}{1-q^{k}}(z ; q)_{k}\right| \leq(-r ;|q|)_{\infty} \sum_{k=1}^{\infty} \frac{|q|^{k}}{1-|q|^{k}} .
$$

The partial sums therefore are a normal family and are uniformly convergent on every compact subset of the complex plane. The limit of these partial sums is $S_{q}(z)$ and is therefore an entire function of the complex variable $z$.

Let $M(r)=\max _{|z| \leq r}\left|S_{q}(z)\right|$. Then

$$
M(r) \leq(-r ;|q|)_{\infty} \sum_{k=1}^{\infty} \frac{|q|^{k}}{1-|q|^{k}}
$$


and $(-r ;|q|)_{\infty}=E_{|q|}(r)$ is the maximum of $E_{|q|}(z)$ on the ball $\{|z| \leq r\}$. The function $E_{q}$ is an entire function of order zero, which can be seen from the coefficients $a_{n}$ of its Taylor series and the formula [2, Theorem 2.2.2]

$$
\limsup _{n \rightarrow \infty} \frac{n \log n}{\log \left(1 /\left|a_{n}\right|\right)}
$$

for the order of $\sum_{n=0}^{\infty} a_{n} z^{n}$. Hence also $S_{q}$ has order zero.

Observe that for $0<q<1$ we have

$$
M(r)=\max _{|z| \leq r}\left|S_{q}(z)\right|=\sum_{k=1}^{\infty} \frac{q^{k}}{1-q^{k}}(-r ; q)_{k}
$$

and some simple bounds give

$$
(q ; q)_{\infty} \sum_{k=1}^{\infty} \frac{q^{k}}{(q ; q)_{k}}(-r ; q)_{k} \leq M(r) \leq(-r ; q)_{\infty} \sum_{k=1}^{\infty} \frac{q^{k}}{1-q^{k}} .
$$

For the lower bound we can use the $q$-binomial theorem (1.3) to find

$$
(-r q ; q)_{\infty}-(q ; q)_{\infty} \leq M(r) \leq(-r ; q)_{\infty} \sum_{k=1}^{\infty} \frac{q^{k}}{1-q^{k}},
$$

which shows that $M(r)$ behaves like $E_{q}(q r)-C_{1} \leq M(r) \leq C_{2} E_{q}(r)$, where $C_{1}$ and $C_{2}$ are constants (which depend on $q$ ).

Euler [8, §§14-15] essentially also stated the following Taylor expansion.

Property 2.2. The $q$-logarithm (1.2) has the following Taylor series around $x=0$ :

$$
S_{q}(x)=-\sum_{k=1}^{\infty} \frac{q^{k}}{1-q^{k}}\left(1+q^{k(k-1) / 2} \frac{(-x)^{k}}{(q ; q)_{k}}\right) .
$$

Proof. Use the $q$-binomial theorem (1.3) with $x=z q^{k}$ and $a=q^{-k}$ to find

$$
(z ; q)_{k}=\sum_{j=0}^{k}\left[\begin{array}{l}
k \\
j
\end{array}\right] q^{j(j-1) / 2}(-z)^{j}, \quad\left[\begin{array}{l}
k \\
j
\end{array}\right]=\frac{(q ; q)_{k}}{(q ; q)_{j}(q ; q)_{k-j}} .
$$

Use this in (1.2), and change the order of summation to find

$$
S_{q}(x)=-\sum_{k=1}^{\infty} \frac{q^{k}}{1-q^{k}}-\sum_{j=1}^{\infty} q^{j(j-1) / 2}(-x)^{j} \sum_{k=j}^{\infty} \frac{q^{k}}{1-q^{k}} \frac{(q ; q)_{k}}{(q ; q)_{j}(q ; q)_{k-j}} .
$$

With a new summation index $k=j+\ell$ this becomes

$$
S_{q}(x)=-\sum_{k=1}^{\infty} \frac{q^{k}}{1-q^{k}}-\sum_{j=1}^{\infty} \frac{q^{j}}{1-q^{j}} q^{j(j-1) / 2}(-x)^{j} \sum_{\ell=0}^{\infty} q^{\ell} \frac{\left(q^{j} ; q\right)_{\ell}}{(q ; q)_{\ell}} .
$$

Now use the $q$-binomial theorem (1.3) to sum over $\ell$ to find

$$
S_{q}(x)=-\sum_{k=1}^{\infty} \frac{q^{k}}{1-q^{k}}-\sum_{j=1}^{\infty} \frac{q^{j}}{1-q^{j}} q^{j(j-1) / 2} \frac{(-x)^{j}}{(q ; q)_{j}} .
$$

If we combine both series, the required expansion follows. 
This result can be written in terms of basic hypergeometric series as

$$
S_{q}(x)=-\frac{q}{1-q}{ }_{2} \phi_{1}\left(\begin{array}{c}
q, q \\
q^{2}
\end{array} ; q, q\right)-\frac{q x}{(1-q)^{2}}{ }_{2} \phi_{2}\left(\begin{array}{c}
q, q \\
q^{2}, q^{2}
\end{array} ; q, q^{2} x\right) .
$$

The growth of the coefficients in this Taylor series again shows that $S_{q}$ is an entire function of order zero if we use the formula (2.1) for the order of $\sum_{n=0}^{\infty} a_{n} z^{n}$; see also [11, §4].

Next we mention the following $q$-integral representation, where we use Jackson's $q$-integral (see [10, §1.11])

$$
\int_{0}^{a} f(t) d_{q} t=(1-q) a \sum_{k=0}^{\infty} f\left(a q^{k}\right) q^{k}
$$

defined for functions $f$ whenever the right-hand side converges.

Property 2.3. For every $x \in \mathbb{C}$ we have

$$
S_{q}(x)=-\frac{q(1-x)}{1-q} \int_{0}^{1} G_{q}(q x, q t) d_{q} t
$$

with

$$
G_{q}(x, t)=\sum_{k=0}^{\infty} t^{k}(x ; q)_{k}={ }_{2} \phi_{1}\left(\begin{array}{c}
x, q \\
0
\end{array} ; q, t\right)=\frac{1}{1-t}{ }_{1} \phi_{1}\left(\begin{array}{c}
q \\
q t
\end{array} ; q, x t\right) .
$$

Since $\int_{0}^{a} f(t) d_{q} t \rightarrow \int_{0}^{a} f(t) d t$ when $q \rightarrow 1$ and $G_{q}(x, t) \rightarrow 1 /(1-t(1-x))$ when $q \rightarrow 1$ for $x>0$, we see (at least formally) that Property 2.3 is a $q$-analogue of the integral representation

$$
\log (x)=-\int_{0}^{1} \frac{1-x}{1-t(1-x)} d t, \quad x \notin(-\infty, 0]
$$

for the logarithm.

Proof. Observe that

$$
\frac{1-q}{1-q^{k+1}}=(1-q) \sum_{p=0}^{\infty} q^{(k+1) p}=\int_{0}^{1} t^{k} d_{q} t .
$$

Inserting this in the definition (1.2) of $S_{q}$ and interchanging summations, which is justified by the absolute convergence of the double sum, give the result. The identity between the basic hypergeometric series representing $G_{q}(x, t)$ is the case $c=0$ of [10, (III.4)].

Note that, as in the proof of Property 2.2, one can show that

$$
G_{q}(x, t)=\sum_{j=0}^{\infty} \frac{(-x t)^{j} q^{j(j-1) / 2}}{(t ; q)_{j+1}} .
$$

\section{THE $q$-DIFFERENCE EQUATION}

The function $S_{q}$ satisfies a simple $q$-difference equation:

Property 3.1. The $q$-logarithm (1.2) satisfies

$$
S_{q}(x / q)-S_{q}(x)=1-(x ; q)_{\infty} .
$$


Proof. Recall the $q$-difference operator

$$
D_{q} f(x)=\frac{f(q x)-f(x)}{x(q-1)} .
$$

Then a simple exercise is

$$
D_{1 / q}(x ; q)_{k}=-\frac{1-q^{k}}{1-q}(x ; q)_{k-1} .
$$

Use this in (1.2) to find

$$
D_{1 / q} S_{q}(x)=\sum_{k=1}^{\infty} \frac{q^{k}}{1-q^{k}} \frac{1-q^{k}}{1-q}(x ; q)_{k-1}=\frac{q}{1-q} \sum_{k=0}^{\infty} q^{k}(x ; q)_{k} .
$$

Observe that $(x ; q)_{k+1}-(x ; q)_{k}=(x ; q)_{k}\left[1-x q^{k}-1\right]=-x q^{k}(x ; q)_{k}$. Summing we find $-x \sum_{k=0}^{n} q^{k}(x ; q)_{k}=(x ; q)_{n+1}-(x ; q)_{0}$, and when $n \rightarrow \infty$,

$$
\sum_{k=0}^{\infty} q^{k}(x ; q)_{k}=\frac{1-(x ; q)_{\infty}}{x} .
$$

If we use this result, then

$$
D_{1 / q} S_{q}(x)=\frac{q}{1-q} \frac{1-(x ; q)_{\infty}}{x},
$$

which is (3.1).

In order to see how this is related to the classical derivative of $\log x$, one may rewrite this as

$$
D_{q}\left((1-q) S_{q}(x)\right)=\frac{1}{x}-\frac{(q x ; q)_{\infty}}{x} .
$$

This $q$-difference equation can already be found in [8, $\S 6]$, where Euler writes $s=$ $S_{q}(x)$ and $t=S_{q}(x / q)$ and gives the relation

$$
1+s-t=(1-x)\left(1-\frac{x}{a}\right)\left(1-\frac{x}{a^{2}}\right)\left(1-\frac{x}{a^{3}}\right)\left(1-\frac{x}{a^{4}}\right)\left(1-\frac{x}{a^{5}}\right) \cdots,
$$

where $q=1 / a$.

As a corollary one has $[8, \S 7]$.

Property 3.2. For every positive integer $n$ one has $S_{q}\left(q^{-n}\right)=n$.

Proof. Use (3.1) with $x=q^{-n+1}$ to find $S_{q}\left(q^{-n}\right)-S_{q}\left(q^{-n+1}\right)=1$, since $(x ; q)_{\infty}$ vanishes whenever $x=q^{-n}$ for $n \geq 0$. The result then follows by induction and $S_{q}(1)=0$.

It is this property, which is quite similar to $\log _{a} a^{n}=n$, where $\log _{a}$ is the logarithm with base $a$, which gives $S_{q}$ the flavor of a $q$-logarithm and which made Euler consider this function as an interpolation of the logarithm; see [11, §1]. Observe that this interpolation property can be stated as follows: $-\log q S_{q}(x)$ approximates $\log x$ as $q \uparrow 1$ and for fixed $q$ this approximation is perfect if $x=q^{-n}(n=1,2, \ldots)$.

Another interesting value is

$$
S_{q}(0)=-\sum_{k=1}^{\infty} \frac{q^{k}}{1-q^{k}}=-\zeta_{q}(1),
$$


which is a $q$-analogue of the harmonic series, where the $q$-analogue of the $\zeta$-function is defined by

$$
\zeta_{q}(s)=\sum_{n=1}^{\infty} \frac{n^{s-1} q^{n}}{1-q^{n}} .
$$

It has been proved (see Erdős [7, Borwein [3, 4, Van Assche [27]) that this quantity is irrational whenever $q=1 / p$ with $p$ an integer $\geq 2$. For the specific argument 1 this coincides, up to a factor, with the value at 1 of the $q$ - $\zeta$-function considered by Ueno and Nishizawa 26 .

The values of $S_{q}\left(q^{n}\right)$ for $n \in \mathbb{N}$ are distinctly different, and for these values we do not get the same flavor as the logarithm.

Property 3.3. For every positive integer $n$ one has

$$
S_{q}\left(q^{n}\right)=-n+(q ; q)_{\infty} \sum_{k=0}^{n-1} \frac{1}{(q ; q)_{k}} .
$$

Proof. Choose $x=q^{k+1}$ in (3.1). Then $S_{q}\left(q^{k}\right)-S_{q}\left(q^{k+1}\right)=1-\left(q^{k+1} ; q\right)_{\infty}$. Summing and using the telescoping property give

$$
S_{q}\left(q^{0}\right)-S_{q}\left(q^{n}\right)=\sum_{k=0}^{n-1}\left(S_{q}\left(q^{k}\right)-S_{q}\left(q^{k+1}\right)\right)=n-\sum_{k=0}^{n-1}\left(q^{k+1} ; q\right)_{\infty} .
$$

By Property 3.2 we have $S_{q}(1)=0$. Now $\left(q^{k+1} ; q\right)_{\infty}=(q ; q)_{\infty} /(q ; q)_{k}$ gives the required expression (3.2).

In order to see how this approximates $\log x$, one may reformulate this as

$$
-\log q S_{q}\left(q^{n}\right)=\log q^{n}-\log q \sum_{k=0}^{n-1}\left(q^{k+1} ; q\right)_{\infty} .
$$

In [8, §10] Euler writes $s=S_{q}\left(q^{n}\right), t=S_{q}\left(q^{n-1}\right), u=S_{q}\left(q^{n-2}\right)$, and he writes the recursion

$$
s=\frac{2 t-u+a q^{n}(1-t)}{1-a q^{n}},
$$

where $q=1 / a$. In contemporary notation we write $y_{n}=S_{q}\left(q^{n}\right)$ and obtain the recurrence relation

$$
y_{n}\left(1-q^{n-1}\right)-\left(2-q^{n-1}\right) y_{n-1}+y_{n-2}=q^{n-1} .
$$

One can verify that this recurrence relation indeed holds for $y_{n}=S_{q}\left(q^{n}\right)$ given in (3.2). More generally one in fact has

$$
(1-q x) S_{q}\left(q^{2} x\right)-(2-q x) S_{q}(q x)+S_{q}(x)=q x,
$$

which is a second order non-homogeneous $q$-difference equation for $S_{q}$.

Note that the explicit evaluation $S\left(q^{-n}\right)=n, n \in \mathbb{N}$, gives the following summation formulas:

$$
\sum_{k=1}^{n} \frac{\left(q^{-n} ; q\right)_{k}}{1-q^{k}} q^{k}=-n, \quad \sum_{k=1}^{\infty} \frac{q^{k(k+1) / 2}(-1)^{k-1} q^{-n k}}{\left(1-q^{k}\right)(q ; q)_{k}}=n+\sum_{k=1}^{\infty} \frac{q^{k}}{1-q^{k}},
$$


using the definition of $S_{q}(x)$ and the Taylor expansion in Property 2.2. Similarly, the evaluation at $q^{n}, n \in \mathbb{N}$, given in (3.2) gives the summation formulas

$$
\begin{aligned}
& \sum_{k=1}^{\infty} \frac{\left(q^{n} ; q\right)_{k}}{1-q^{k}} q^{k}=n-\sum_{k=0}^{n-1}\left(q^{k+1} ; q\right)_{\infty} \\
& \sum_{k=1}^{\infty} \frac{q^{k(k+1) / 2}(-1)^{k-1} q^{n k}}{\left(1-q^{k}\right)(q ; q)_{k}}=-n+\sum_{k=1}^{\infty} \frac{q^{k}}{1-q^{k}}+\sum_{k=0}^{n-1}\left(q^{k+1} ; q\right)_{\infty}
\end{aligned}
$$

Note that all infinite series are absolutely convergent and that for $n=0$ the results in (3.3) and (3.4) coincide. The first sums become trivial, and the second sum gives the following expansion for $\zeta_{q}(1)$ :

$$
\zeta_{q}(1)=\sum_{k=1}^{\infty} \frac{q^{k}}{1-q^{k}}=\sum_{k=1}^{\infty} \frac{q^{k(k+1) / 2}(-1)^{k-1}}{\left(1-q^{k}\right)(q ; q)_{k}} .
$$

Using (3.5) in Property 2.2 gives the expansion

$$
S_{q}(x)=-\sum_{k=1}^{\infty} \frac{q^{k(k+1) / 2}(-1)^{k-1}\left(1-x^{k}\right)}{\left(1-q^{k}\right)(q ; q)_{k}},
$$

so that in particular

$$
-\frac{d S_{q}}{d x}(1)=\lim _{x \rightarrow 1} \frac{S_{q}(x)}{1-x}=-\sum_{k=1}^{\infty} \frac{k q^{k(k+1) / 2}(-1)^{k-1}}{\left(1-q^{k}\right)(q ; q)_{k}} .
$$

\section{An EXTENSiOn OF THE $q$-LOGARITHM AND LAMBERT SERIES}

If we have the definition of $S_{q}(x)$ resembling Lambert series, it is natural to look for the extension

$$
F_{q}(x, t)=-\sum_{k=1}^{\infty}(x ; q)_{k} \frac{t^{k}}{1-t^{k}}
$$

which is a Lambert series; see [15, §58.C]. Since $\left|(x ; q)_{k}\right| \leq(-|x| ;|q|)_{k} \leq(-r ;|q|)_{\infty}$ for $x$ in $\{x \in \mathbb{C}|| x \mid \leq r\}$, the convergence in (4.1) is uniform on compact sets in $x$ and on compact subsets of the open unit disk in $t$. Also since the series $-\sum_{k=1}^{\infty}(x ; q)_{k} t^{k}$ is absolutely convergent for $|t|<1$ uniformly in $x$ in compact sets, it follows by [15, Satz 259] that $F_{q}$ is analytic for $(x, t) \in \mathbb{C} \times\{t \in \mathbb{C}|| t \mid<1\}$. Observe that $S_{q}(x)=F_{q}(x, q)$.

The general theory of Lambert series then gives the power series of $F$ in powers of $t$ :

$$
F_{q}(x, t)=\sum_{\ell=1}^{\infty}\left(\sum_{k \mid \ell}(x ; q)_{k}\right) t^{\ell} \Longrightarrow S_{q}(x)=\sum_{\ell=1}^{\infty}\left(\sum_{k \mid \ell}(x ; q)_{k}\right) q^{\ell}
$$

We are mainly interested in the power series development with respect to $x$.

Property 4.1. For $|t|<1$ one has

$$
F_{q}(x, t)=-\sum_{k=1}^{\infty} \frac{t^{k}}{1-t^{k}}-\sum_{\ell=1}^{\infty} x^{\ell}(-1)^{\ell} q^{\ell(\ell-1) / 2}\left(\sum_{n=1}^{\infty} t^{n \ell} \frac{\left(t^{n} q^{\ell+1} ; q\right)_{\infty}}{\left(t^{n} ; q\right)_{\infty}}\right)
$$


In case $t=q$, Property 4.1 reduces to Property 2.2. and this is equivalent to the summation formula

$$
\sum_{n=1}^{\infty} q^{n \ell} \frac{\left(q^{\ell+n+1} ; q\right)_{\infty}}{\left(q^{n} ; q\right)_{\infty}}=\frac{q^{\ell}}{\left(1-q^{\ell}\right)(q ; q)_{\ell}} \Longrightarrow \sum_{n=1}^{\infty} \frac{(q ; q)_{n-1}}{\left(q^{\ell+1} ; q\right)_{n}} q^{n \ell}=\frac{q^{\ell}}{1-q^{\ell}}
$$

for $\ell \in \mathbb{N}, \ell \geq 1$. This can be obtained as a special case of the $q$-Gauss sum [10, (1.5.1)].

Proof. The proof is along the same lines as the proof of Property 2.2 We find similarly

$$
F_{q}(x, t)=-\sum_{k=1} \frac{t^{k}}{1-t^{k}}-\sum_{j=1}^{\infty} q^{j(j-1) / 2}(-x t)^{j} \sum_{\ell=0}^{\infty} \frac{\left(q^{j+1} ; q\right)_{\ell}}{(q ; q)_{\ell}} \frac{t^{\ell}}{1-t^{j+\ell}}
$$

and we write

$$
\begin{aligned}
\sum_{\ell=0}^{\infty} \frac{\left(q^{j+1} ; q\right)_{\ell}}{(q ; q)_{\ell}} \frac{t^{\ell}}{1-t^{j+\ell}} & =\sum_{\ell=0}^{\infty} \frac{\left(q^{j+1} ; q\right)_{\ell}}{(q ; q)_{\ell}} t^{\ell} \sum_{p=0}^{\infty} t^{p(j+\ell)} \\
& =\sum_{p=0}^{\infty} t^{j p} \sum_{\ell=0}^{\infty} \frac{\left(q^{j+1} ; q\right)_{\ell}}{(q ; q)_{\ell}} t^{\ell(1+p)}=\sum_{p=0}^{\infty} t^{j p} \frac{\left(t^{1+p} q^{j+1} ; q\right)_{\infty}}{\left(t^{1+p} ; q\right)_{\infty}}
\end{aligned}
$$

using the $q$-binomial theorem again and the absolute convergence of the double sum, which justifies the interchange of summations. Using this and replacing $n=p+1$ we get the result.

Consider the case $t=q^{2}$. Following the line of proof of Property 2.2 we write

$$
-\sum_{k=1}^{\infty} \frac{q^{2 k}(x ; q)_{k}}{1-q^{2 k}}=-\sum_{k=1}^{\infty} \frac{q^{2 k}}{1-q^{2 k}}-\sum_{j=1}^{\infty} \frac{(-1)^{j} q^{j(j-1) / 2} x^{j}}{(q ; q)_{j}} \sum_{\ell=0}^{\infty} \frac{(q ; q)_{\ell+j} q^{2 \ell+2 j}}{(q ; q)_{\ell}\left(1-q^{2 \ell+2 j}\right)}
$$

and we can write the inner sum over $\ell$ as

$$
\sum_{\ell=0}^{\infty} \frac{(q ; q)_{\ell+j-1} q^{2 \ell+2 j}}{(q ; q)_{\ell}\left(1+q^{\ell+j}\right)}=\frac{(q ; q)_{j-1} q^{2 j}}{1+q^{j}} \sum_{\ell=0}^{\infty} \frac{\left(q^{j} ; q\right)_{\ell}\left(-q^{j} ; q\right)_{\ell}}{(q ; q)_{\ell}\left(-q^{j+1} ; q\right)_{\ell}} q^{2 \ell} .
$$

Using Property 4.1 for $t=q^{2}$ then gives

$$
\sum_{n=1}^{\infty} q^{2 n j} \frac{\left(q^{2 n+j+1} ; q\right)_{\infty}}{\left(q^{2 n} ; q\right)_{\infty}}=\frac{q^{2 j}}{\left(1-q^{2 j}\right)} \sum_{\ell=0}^{\infty} \frac{\left(q^{j} ; q\right)_{\ell}\left(-q^{j} ; q\right)_{\ell}}{(q ; q)_{\ell}\left(-q^{j+1} ; q\right)_{\ell}} q^{2 \ell} .
$$

This can also be proved directly using the $q$-binomial theorem and geometric series. We can rewrite (4.3) in standard basic hypergeometric series form (see [10]) as the quadratic transformation

$$
\frac{\left(1-q^{2 j}\right)}{\left(q^{2} ; q\right)_{j+1}}{ }_{3} \phi_{2}\left(\begin{array}{c}
q^{2}, q^{2}, q^{3} \\
q^{j+3}, q^{j+4}
\end{array} ; q^{2}, q^{2}\right)={ }_{2} \phi_{1}\left(\begin{array}{c}
q^{j},-q^{j} \\
-q^{j+1}
\end{array} ; q, q^{2}\right) .
$$

Analogous to Property 2.3 and using the notation of Property 2.3, we have the following.

Property 4.2. For $|p|<1$ one has

$$
F_{q}(x, p)=\frac{-p(1-x)}{(1-p)} \int_{0}^{1} G(q x, p t) d_{p} t
$$




\section{A $q$-Analogue of the Dilogarithm}

Euler's dilogarithm is defined by the first equality in

$$
\operatorname{Li}_{2}(x)=\sum_{n=1}^{\infty} \frac{x^{n}}{n^{2}}=-\int_{0}^{x} \frac{\log (1-t)}{t} d t=-\int_{1-x}^{1} \frac{\log (t)}{1-t} d t=\frac{\pi^{2}}{6}-\operatorname{Li}_{2}(1-x)
$$

for $0 \leq x \leq 1$; see [18, [14 for more information and references. Here we use $\operatorname{Li}_{2}(1)=\zeta(2)=\frac{\pi^{2}}{6}$. In particular, $x \frac{d \mathrm{Li}_{2}}{d x}=-\log (1-x)$, and the definition by the series can be extended to complex $x$ being absolutely convergent for $|x| \leq 1$.

We define the $q$-dilogarithm by

$$
\operatorname{Li}_{2}(x ; q)=\sum_{k=1}^{\infty} \frac{q^{k}}{\left(1-q^{k}\right)^{2}}(x ; q)_{k} .
$$

We have $\lim _{q \uparrow 1}(1-q)^{2} \operatorname{Li}_{2}(x ; q)=\sum_{k=1}^{\infty}(1-x)^{k} / k^{2}=\operatorname{Li}_{2}(1-x)$. In this case we can justify the interchange of the limit and summation using dominated convergence. We assume $0<q<1$, and we first observe that $\left|(x ; q)_{k}\right| \leq 1$ for $|1-x| \leq 1$. Next we use

$$
\frac{1-q^{k}}{1-q}=\sum_{j=0}^{k-1} q^{j}=q^{(k-1) / 2} \begin{cases}\sum_{j=0}^{\frac{k}{2}-1}\left(q^{j+\frac{1}{2}}+q^{-j-\frac{1}{2}}\right), & k \text { even, } \\ 1+\sum_{j=0}^{\frac{k-1}{2}-1}\left(q^{j+1}+q^{-j-1}\right), & k \text { odd },\end{cases}
$$

and $x+1 / x \geq 2$ for $x \in[0,1]$ then gives

$$
\frac{1-q^{k}}{1-q} \geq k q^{(k-1) / 2}
$$

so that

$$
q^{k} \frac{(1-q)^{2}}{\left(1-q^{k}\right)^{2}} \leq \frac{1}{k^{2}}
$$

Combining both estimates gives

$$
\left|\frac{q^{k}}{\left(1-q^{k}\right)^{2}}(x ; q)_{k}\right| \leq \frac{1}{k^{2}}
$$

for $|1-x| \leq 1$, and dominated convergence is established.

We list some properties of the $q$-dilogarithm. In the following we use $\zeta_{q}(2)=$ $\sum_{k=1}^{\infty} \frac{q^{k}}{\left(1-q^{k}\right)^{2}}$ as an analogue of $\frac{1}{6} \pi^{2}$. This is equal to the $q$ - $\zeta$-function

$$
\zeta_{q}(s)=\sum_{n=1}^{\infty} \frac{n^{s-1} q^{n}}{1-q^{n}}
$$

for $s=2$ since

$$
\sum_{n=1}^{\infty} \frac{n q^{n}}{1-q^{n}}=\sum_{n=1}^{\infty} \sum_{k=1}^{\infty} n q^{n k}=\sum_{k=1}^{\infty} \frac{q^{k}}{\left(1-q^{k}\right)^{2}}
$$

(see, e.g., 21, Part VIII, Chapter 1, problem 75]). This quantity was considered by Zudilin [28, 29], Krattenthaler et al. [17, Postelmans and Van Assche 22], who studied its irrationality when $1 / q$ is an integer $\geq 2$. Note that this no longer corresponds to Ueno and Nishizawa [26, who essentially have $\sum_{k=1}^{\infty} \frac{q^{2 k}}{\left(1-q^{k}\right)^{2}}$ as the value at 2 for their $q$ - $\zeta$-function. 
Property 5.1. $\operatorname{Li}_{2}(\cdot ; q)$ is an entire function of order zero. Moreover, we have the special values

$$
\operatorname{Li}_{2}(1 ; q)=0, \quad \operatorname{Li}_{2}(0 ; q)=\zeta_{q}(2), \quad \operatorname{Li}_{2}\left(q^{-n} ; q\right)=-\sum_{k=1}^{n} \frac{k}{1-q^{k}},
$$

and $(1-q)(1-x)\left(D_{q} \operatorname{Li}_{2}(\cdot ; q)\right)(x)=S_{q}(x)$ and

$$
\operatorname{Li}_{2}(x ; q)=\zeta_{q}(2)+\frac{1}{1-q} \int_{0}^{x} \frac{S_{q}(t)}{1-t} d_{q} t .
$$

Moreover, the $q$-dilogarithm has the Taylor expansion

$$
\operatorname{Li}_{2}(x ; q)=\zeta_{q}(2)+\sum_{j=1}^{\infty} \frac{(-1)^{j} q^{j(j+1) / 2} x^{j}}{\left(1-q^{j}\right)^{2}}{ }_{2} \phi_{1}\left(\begin{array}{c}
q^{j}, q^{j} \\
q^{j+1}
\end{array} ; q, q\right) .
$$

Here the ${ }_{2} \phi_{1}$-series is defined by

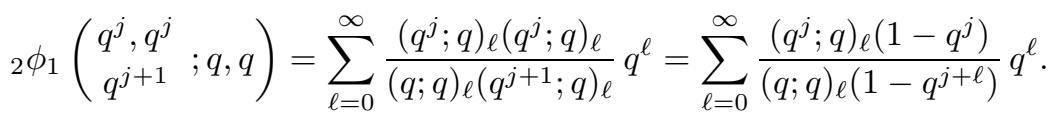

Unfortunately, this series cannot be summed using the (non-terminating) $q$-ChuVandermonde sum.

Note that after multiplying the integral representation for $\operatorname{Li}_{2}(x ; q)$ by $(1-q)^{2}$, we can take a formal limit $q \uparrow 1$ to get

$$
\operatorname{Li}_{2}(1-x)=\frac{\pi^{2}}{6}+\int_{0}^{x} \frac{\log (t)}{1-t} d t=-\int_{0}^{1-x} \frac{\log (1-t)}{t} d t
$$

so that we recover the integral representation for the dilogarithm.

Proof. The proof of $\operatorname{Li}_{2}(\cdot ; q)$ being an entire function of order zero is derived as in Property 2.1. Since $(q x ; q)_{k}-(x ; q)_{k}=x\left(1-q^{k}\right)(q x ; q)_{k-1}$ we obtain

$$
\operatorname{Li}_{2}(q x ; q)-\mathrm{Li}_{2}(x ; q)=\frac{x}{1-x} \sum_{k=1}^{\infty} \frac{q^{k}(x ; q)_{k}}{1-q^{k}}=\frac{-x}{1-x} S_{q}(x) .
$$

This implies $(1-q)(1-x)\left(D_{q} \operatorname{Li}_{2}(\cdot ; q)\right)(x)=S_{q}(x)$.

Using (5.2) for $x=q^{-n}, n \in \mathbb{N}$, and $\operatorname{Li}_{2}(1 ; q)=0, S\left(q^{-n}\right)=n$, we find the value for $\operatorname{Li}_{2}\left(q^{-n} ; q\right)$. Iterating (5.2) we get

$$
\operatorname{Li}_{2}(x ; q)=\sum_{k=0}^{N} \frac{x q^{k}}{1-x q^{k}} S_{q}\left(x q^{k}\right)+\operatorname{Li}_{2}\left(x q^{N+1} ; q\right),
$$

and by letting $N \rightarrow \infty$ we get the convergent series expansion

$$
\operatorname{Li}_{2}(x ; q)=\operatorname{Li}_{2}(0 ; q)+\sum_{k=0}^{\infty} \frac{x q^{k}}{1-x q^{k}} S_{q}\left(x q^{k}\right)=\zeta_{q}(2)+\frac{1}{1-q} \int_{0}^{x} \frac{S_{q}(t)}{1-t} d_{q} t .
$$

Finally, the Taylor expansion proceeds as in the proof of Property 2.2. and we find

$$
\operatorname{Li}_{2}(x ; q)=\sum_{k=1}^{\infty} \frac{q^{k}}{\left(1-q^{k}\right)^{2}}+\sum_{j=1}^{\infty} \frac{(-x)^{j} q^{j(j-1) / 2}}{(q ; q)_{j}} \sum_{\ell=0}^{\infty} \frac{(q ; q)_{j+\ell} q^{j+\ell}}{(q ; q)_{\ell}\left(1-q^{j+\ell}\right)^{2}} .
$$


The inner sum over $\ell$ can be rewritten as

$$
\frac{q^{j}(q ; q)_{j-1}}{1-q^{j}} \sum_{\ell=0}^{\infty} \frac{\left(q^{j} ; q\right)_{\ell}\left(q^{j} ; q\right)_{\ell}}{(q ; q)_{\ell}\left(q^{j+1} ; q\right)_{\ell}} q^{\ell}
$$

and this gives the result.

The evaluation of the $q$-dilogarithm gives the following summation (cf. (3.3) ):

$$
\begin{aligned}
\sum_{k=1}^{n} \frac{\left(q^{-n} ; q\right)_{k} q^{k}}{\left(1-q^{k}\right)^{2}} & =-\sum_{k=1}^{n} \frac{k}{1-q^{k}} \\
= & \sum_{j=1}^{\infty} \frac{q^{j}}{\left(1-q^{j}\right)^{2}}+\sum_{j=1}^{\infty} \frac{(-1)^{j} q^{j(j+1) / 2} q^{-n j}}{\left(1-q^{j}\right)^{2}}{ }_{2} \phi_{1}\left(\begin{array}{c}
q^{j}, q^{j} \\
q^{j+1}
\end{array} ; q, q\right) .
\end{aligned}
$$

In particular, for $n=0$ we obtain an alternating series representation for $\zeta_{q}(2)$ :

$$
\zeta_{q}(2)=\sum_{j=1}^{\infty} \frac{(-1)^{j-1} q^{j(j+1) / 2}}{\left(1-q^{j}\right)^{2}}{ }_{2} \phi_{1}\left(\begin{array}{c}
q^{j}, q^{j} \\
q^{j+1}
\end{array} ; q, q\right) .
$$

If we write $\operatorname{Li}_{2}(x ; q)=\sum_{n=0}^{\infty} a_{n} x^{n}, S_{q}(x)=\sum_{n=0}^{\infty} b_{n} x^{n}$ temporarily, then (5.2) implies that $q^{n} a_{n}-a_{n}$ equals the coefficient, say $c_{n}$, of $x^{n}$ in $-S_{q}(x) x /(1-x)$. If we use $-x /(1-x)=\sum_{k=1}^{\infty}-x^{k}$, it follows that $c_{n}=-\sum_{p=0}^{n-1} b_{p}$. Note that the relation is trivial in case $n=0$, and for integer $n \geq 1$ we find from the explicit Taylor expansions for $S_{q}(\cdot)$ and $\operatorname{Li}_{2}(\cdot ; q)$ the relation

$$
\frac{(-1)^{n-1} q^{n(n+1) / 2}}{\left(1-q^{n}\right)}{ }_{2} \phi_{1}\left(\begin{array}{c}
q^{n}, q^{n} \\
q^{n+1}
\end{array} ; q, q\right)=\sum_{k=1}^{\infty} \frac{q^{k}}{1-q^{k}}+\sum_{j=1}^{n-1} \frac{(-1)^{j} q^{j(j+1) / 2}}{\left(1-q^{j}\right)(q ; q)_{j}}
$$

Note that this relation gives an explicit expression for the remainder if approximating $\zeta_{q}(1)$ with the alternating series as in (3.5). Of course, we get the same result if we use the Taylor expansion of $S_{q}$ as in Property 2.2 in the integral representation for the $q$-dilogarithm in Property 5.1 .

The classical dilogarithm satisfies many interesting properties, such as a simple functional equation, a five-term recursion, a characterisation by these first two properties, explicit evaluation at certain special points, etc.; see [18, [14] for more information and references. It would be interesting to see if these interesting properties have appropriate analogues for the $q$-analogue of the dilogarithm discussed here.

\section{OTHER $q$-LOGARITHMS}

In the physics literature (see e.g. [25]), one defines $\ln _{q}(x)=\frac{x^{1-q}-1}{1-q}$. There are no $q$-series, $q$-Pochhammer symbols, $q$-difference relations, etc. The choice of the letter $q$ and the fact that $\lim _{q \rightarrow 1} \ln _{q}(x)=\log x$ are not sufficient motivation to call this a $q$-analogue. It just shows that the logarithmic function is somewhere between the constant function and powers $x^{\alpha}-1$ for $\alpha>0$. This $q$-analogue of the logarithm plays a role in statistical mechanics and, as pointed out in [13], was also introduced by Euler in 1779; see [13] for more information and references.

Borwein [4, Zudilin et al. [19], and Van Assche [27] consider

$$
\ln _{q}(1+z)=\sum_{k=1}^{\infty} \frac{(-1)^{k} z^{k}}{1-q^{k}}, \quad|z|<|q|
$$


with $|q|>1$. They prove that $\ln _{q}(1+z)$ is irrational for $z= \pm 1$ and $q$ an integer greater than 2. For $z=-1$ one has a $q$-analogue of the harmonic series, and this is essentially the generating function of $d_{n}=\sum_{k \mid n} 1$, i.e. the number of divisors of $n$. A similar formula, but now for $0<q<1$,

$$
\log _{q}(z)=\sum_{k=1}^{\infty} \frac{z^{n}}{1-q^{n}}=\frac{z e_{q}^{\prime}(z)}{e_{q}(z)}, \quad|z|<1,
$$

has been considered as a $q$-analogue of the logarithm by Kirillov [14] and Koornwinder [16]. This $q$-analogue is well adapted to non-commutative algebras; see [14, §2.5, Ex. 11], [16, Prop. 6.1], since $\log _{q}(x+y-x y)=\log _{q}(x)+\log _{q}(y)$ for $x y=q y x$. The corresponding $q$-analogue of the dilogarithm, provisionally denoted by $\widetilde{\operatorname{Li}_{2}}(x ; q)$, is defined by

$$
\widetilde{\operatorname{Li}_{2}}(x ; q)=\sum_{k=1}^{\infty} \frac{z^{k}}{k\left(1-q^{k}\right)}=\log \left(e_{q}(z)\right) \Longrightarrow \log _{q}(z)=z{\widetilde{\operatorname{Li}_{2}}}^{\prime}(z ; q) .
$$

Zudilin [29] considers a similar $q$-logarithm but a different $q$-dilogarithm,

$$
L_{1}(x ; q)=\sum_{n=1}^{\infty} \frac{(x q)^{n}}{1-q^{n}}, \quad L_{2}(x ; q)=\sum_{n=1}^{\infty} \frac{n(x q)^{n}}{1-q^{n}}
$$

and mainly studies simultaneous rational approximation to $L_{1}$ and $L_{2}$ in order to obtain quantitative linear independence over $\mathbb{Q}$ for certain values of these functions.

Other $q$-logarithms are defined as inverses of $q$-exponential functions; see Nelson and Gartley [20] for two different cases viewed from complex function theory, and Chung et al. [5], where implicitly $q$-commuting variables are used. Fock and Goncharov [9, 12 introduce a $q$-logarithm of $\ln \left(e^{z}+1\right)$ by an integral. The corresponding $q$-dilogarithm is essentially Ruijsenaars' hyperbolic $\Gamma$-function; see [23, II.A]. For other $q$-logarithms based on Jacobi theta functions, see Sauloy [24] and Duval [6], where the $q$-logarithms play a role in difference Galois theory in constructing the analogue of a unipotent monodromy representation.

\section{ACKNOWLEDGEMENTS}

This paper was triggered by a lecture on the 1734 letter of Leonhard Euler to Daniel Bernoulli by Walter Gautschi; see [11. We thank Walter Gautschi for useful discussions and for providing us with a translation of [8] (the translation can be downloaded from the E190 page of the Euler archive at http://www .math. dartmouth.edu/ euler). We thank the referee for useful comments, and Hans Haubold for pointing out [13. The first author's work for this paper was mainly done at Technische Universiteit Delft.

\section{REFERENCES}

1. G. E. Andrews, R. Askey, R. Roy, Special Functions, Encyclopedia of Mathematics and its Applications 71, Cambridge University Press, 1999. MR1688958 (2000g:33001)

2. R. P. Boas, Jr., Entire Functions, Academic Press, New York, 1954. MR.0068627 (16:914f)

3. P. Borwein, On the irrationality of $\sum \frac{1}{q^{n}+r}$, J. Number Theory 37 (1991), 253-259. MR.1096442 (92b:11046) 
4. P. Borwein, On the irrationality of certain series, Math. Proc. Cambridge Philos. Soc. 112 (1992), 141-146. MR1162938 (93g:11074)

5. K-S. Chung, W-S. Chung, S-T. Nam, H-J. Kang, New q-derivative and q-logarithm, Internat. J. Theor. Phys. 33 (1994), 2019-2029. MR1306795 (95i:33018)

6. A. Duval, Une remarque sur les "logarithmes" associés à certains caractères, Aequationes Math. 68 (2004), no. 1-2, 88-97. MR2167011 (2006e:39029)

7. P. Erdős, On arithmetical properties of Lambert series, J. Indian Math. Soc. (N.S.) 12 (1948), 63-66. MR0029405 (10:594c)

8. L. Euler, Consideratio quarumdam serierum quae singularibus proprietatibus sunt praeditae, Novi Commentarii Academiae Scientiarum Petropolitanae 3 (1750-1751), pp. 10-12, 86-108; Opera Omnia, Ser. I, Vol. 14, B.G. Teubner, Leipzig, 1925, pp. 516-541.

9. V.V. Fock, A.B. Goncharov, The quantum dilogarithm and representations of quantum cluster varieties, arXiv:math/0702397v6, to appear in Invent. Math.

10. G. Gasper, M. Rahman, Basic Hypergeometric Series, 2nd ed., Encyclopedia of Mathematics and its Applications 96, Cambridge University Press, 2004. MR2128719 (2006d:33028)

11. W. Gautschi, On Euler's attempt to compute logarithms by interpolation: A commentary to his letter of February 17, 1734, to Daniel Bernoulli, J. Comput. Appl. Math. 219 (2008), no. $2,408-415$.

12. A.B. Goncharov, The pentagon relation for the quantum dilogarithm and quantized $M_{0,5}$, arXiv:0706.4054v2

13. G. Kaniadakis, M. Lissia, Editorial, Physica A 340 (2004), xv-xix.

14. A.N. Kirillov, Dilogarithm Identities, Progr. Theoret. Phys. Suppl. 118 (1995), 61-142 (Lectures in Math. Sci. 7, Univ. of Tokyo, 1995). MR1356515 (96h:11102)

15. K. Knopp, Theorie und Anwendung der Unendlichen Reihen, 4th ed., Springer-Verlag, BerlinHeidelberg, 1947. MR0028430 (10:446a)

16. T.H. Koornwinder, Special functions and q-commuting variables, pp. 131-166 in Special Functions, $q$-Series and Related Topics (eds. M.E.H. Ismail, D.R. Masson and M. Rahman), Fields Inst. Commun. 14, Amer. Math. Soc., Providence, RI, 1997. MR1448685 (99e:33024)

17. C. Krattenthaler, T. Rivoal, W. Zudilin, Séries hypergéométriques basiques, q-analogues des valeurs de la fonction zêta et séries d'Eisenstein, J. Inst. Math. Jussieu 5 (2006), 53-79. MR 2195945 (2006k:11137)

18. L. Lewin, Dilogarithms and Associated Functions, Macdonald, London, 1958. MR0105524 $(21: 4264)$

19. T. Matala-Aho, K. Väänänen, W. Zudilin, New irrationality measures for q-logarithms, Math. Comp. 75 (2006), no. 254, 879-889. MR2196997(2007e:11082)

20. C.A. Nelson, M.G. Gartley, On the two q-analogue logarithmic functions: $\ln _{q}(w), \ln \left\{e_{q}(z)\right\}$, J. Phys. A 29 (1996), no. 24, 8099-8115. MR1446909 (98d:33007)

21. G. Pólya, G. Szegö, Problems and Theorems in Analysis, Volume II, Springer-Verlag, New York-Heidelberg, 1976 (revised and enlarged translation of Aufgaben und Lehrsätze aus der Analysis II, 4th edition, 1971). MR0465631 (57:5529)

22. K. Postelmans, W. Van Assche, Irrationality of $\zeta_{q}(1)$ and $\zeta_{q}(2)$, J. Number Theory 126 (2007), 119-154. MR2348015

23. S.N.M. Ruijsenaars, First order analytic difference equations and integrable quantum systems, J. Math. Phys. 38 (1997), no. 2, 1069-1146. MR.1434226 (98m:58065)

24. J. Sauloy, Systèmes aux q-différences singuliers réguliers: Classification, matrice de connexion et monodromie, Ann. Inst. Fourier (Grenoble) 50 (2000), no. 4, 1021-1071. MR1799737 (2001m:39043)

25. C. Tsallis, Possible generalization of Boltzmann-Gibbs statistics, J. Stat. Phys. 52 (1988), 479-487. MR968597 (89i:82119)

26. K. Ueno, M. Nishizawa, Quantum groups and zeta-functions, pp. 115-126 in Quantum Groups (eds. J. Lukierski, Z. Popowicz and J. Sobczyk), PWN, Warsaw, 1995. MR.1647965 (99k:11136)

27. W. Van Assche, Little q-Legendre polynomials and irrationality of certain Lambert series, The Ramanujan Journal 5 (2001), 295-310. MR1876702(2002k:11124) 
28. V.V. Zudilin, On the irrationality measure of the q-analogue of $\zeta(2)$, Mat. Sbornik 193 (2002), no. 8, 49-70 (in Russian); Sbornik Math. 193 (2002), no. 7-8, 1151-1172. MR 1934544 (2003g:11077)

29. W. Zudilin, Approximations to q-logarithms and q-dilogarithms, with applications to q-zeta values, Zap. Nauchn. Sem. POMI 322 (2005), 107-124 (in Russian); J. Math. Sci. (N.Y.) 137 (2006), no. 2, 4673-4683. MR.2138454 (2006h:11088)

IMAPP, FNWI, Radboud Universiteit, Toernooiveld 1, 6525 ED Nijmegen, The NetheRLANDS

E-mail address: e.koelink@math.ru.nl

Departement Wiskunde, Katholieke Universiteit Leuven, Celestijnenlaan 200B, B-3001 Leuven, Belgium

E-mail address: walter@wis.kuleuven.be 